\title{
Prevalence of thyroid dysfunction in diabetic patients
}

\begin{abstract}
Diabetes mellitus and thyroid dysfunction are two major highly-spread health problems worldwide. Both of them affect each other, and the recognition of the relationship between diabetes mellitus and thyroid dysfunction is of importance to guide clinicians in good management of both of them. The present study aimed to find out the prevalence of thyroid dysfunction among diabetic patients in Qurayat Diabetes Center (QDC).
\end{abstract}

Materials and methods: Two hundred (100 type $1 \& 100$ type 2 ) diabetic patients were retrospectively investigated who were admitted for the Diabetes Centre in Al Qurayyat Governorate, Al-Jouf, and KSA. Parameters investigated in the study are age, body mass index, fasting bood glucose, post-prandial blood glucose, glycosylated hemoglobin, free triidothyronine (fT3), free thyroxine (fT4) and thyroid-stimulating hormone (TSH).

Results: Among the diabetic patients studied, 69\% had normal thyroid profile (euthyroid) and $31 \%$ showed thyroid dysfunction ( $25 \%$ had subclinical hypothyroidism, $3.5 \%$ had clinical hypothyroidism, and $2.5 \%$ had clinical hyperthyroidism) while no cases of subclinical hyperthyroidism were found.

Conclusion and recommendation: Subclinical hypothyroidism is the most prevalent thyroid dysfunction in our diabetic patients. It increases the risk of cardiovascular complications, and its most important consequence is high possibility of progression to clinical hypothyroidism. It is therefore important to diagnose thyroid dysfunction in diabetic patients to prevent further complications, and this practice should be carried out in clinical settings.

Keywords: prevalence, diabetes mellitus, hypothyroidism, ft3, ft4, TSH, thyroid, patient, dm, td
Volume 4 Issue 2 - 2017

\author{
Ibrahim M Elmenshawi, Sultan S Alotaibi, \\ Amal Saleh Alazmi, Alhanouf Mamluh Alazmi, \\ Fandiyyah Rajeh Alruwaili, Noor Naif Alazmi, \\ Zuhur Naif Alazmi \\ Cairo University, Egypt
}

Correspondence: Ibrahim M Elmenshawi, Cairo University, Cairo city, Egypt, Tel 0096655327 5280, Email drmenshawi@aol.com

Received: August 31, 2016 | Published: April 03, 2017
Abbreviations: TSH, thyroid-stimulating hormone; DM, diabetes mellitus; TD, thyroid dysfunction; WHO, world health organization; T1DM, type 1 diabetes; T2DM, type 2 diabetes; QDC, qurayat diabetes centre; FBS, fasting blood sugar; PPBS, postprandial blood sugar

\section{Introduction}

The two most common endocrine disorders diagnosed and found in clinical practice in different ages and different populations all the time are diabetes mellitus (DM) and thyroid dysfunction (TD). Both of them affect each other. ${ }^{1}$ All over the world, there is a huge number of people who die because of diabetes. ${ }^{2}$ The world health organization (WHO) indicated that in 2000, patients with DM represented $2.8 \%$ and they predicted that they may reach to about $4.4 \%$ in 2030 . In numbers, the increasing prevalence of diabetes becomes clearer when we know that the number of diabetic patients may raise from 171 million in 2000 to reach 366 million in $2030 .^{3}$ This growing number of diabetic subjects and the complications that they usually suffer from as a result of DM, especially thyroid dysfunction, need more and more research to stop progression of that killer disease.

Diabetes is manifested as a group of metabolic disorders with a common phenotype of increased blood glucose level (hyperglycemia). Hyperglycemia is a disorder of the endocrine system that can be easily described briefly as reduced insulin secretion, compromised insulin action, increased production and decreased breakdown of blood sugar. ${ }^{4}$ Many factors contribute to this highly-spread disease, like obesity, sedentary life-style and high blood pressure. ${ }^{5}$ The pathogenic processes involved in hyperglycemia include autoimmune destruction of pancreatic insulin-secreting cells (3-cells) resulting in insulin deficiency and abnormalities that result in insulin resistance. Diabetes mellitus is divided into two major types; the first is called insulindependent diabetes or type 1 diabetes (T1DM). In this case, pancreatic beta cells release insufficient amounts of insulin or no insulin. The other major type of diabetes is called insulin non-dependent diabetes or type 2 diabetes (T2DM) which is the more common form of DM (90-95\% of all diabetic cases). This type results from combination of resistance to insulin action in body cells and an inadequate insulin secretion from pancreatic beta cells. ${ }^{6}$

In the general population, approximately $6 \%$ of people have some form of TD. However, the prevalence of TD increases to over $10 \%$ in people with diabetes. ${ }^{7}$ The hormones of thyroid gland regulate metabolism processes of carbohydrate in the body and, on the other hand, DM affects thyroid profile. Found together, both of 'ID and $\mathrm{DM}$, especially in uncontrolling of diabetes, causes many health problems. Increased frequency of hypoglycemia in hypothyroidism and development of potentially life-threatening ketoacidosis in thyrotoxicosis are the most serious effects. Despite that, the diagnosis of TD in diabetics takes little consideration. ${ }^{8}$ In diabetic patients, TD may go undiagnosed because of the common signs and symptoms of both disorders. For this reason, TD may be overlooked or attributed to other medical disorders. ${ }^{1}$

There are two types of thyroid dysfunction. One of them is called hypothyroidism which is the most common form of TD in adulthood; it is the case of insufficient production of thyroid hormones (T3 \& T4). The other less-common type is called hyperthyroidism, it is a condition in which thyroid gland releases increased amounts of both 
hormones. Hyperthyroidism speeds up the rate of body metabolism, so it results in sudden loss of body weight, rapid heart-beats, nervousness and increased perspiration. ${ }^{9-10}$

\section{Objective}

The main objective of the current study was to investigate the prevalence of thyroid dysfunction in diabetic patients who are following up at Qurayat Diabetes Centre (QDC) which Located at A1-Qurayyat Governorate, Al-Jouf, Saudi Arabia.

\section{Materials and methods}

The current study involved a retrospective investigation of 200 confirmed diabetic patients of both sexes; males and females (100 males and 100 females). Patients enrolled in the current study involved those patients who were follow up at QDC in Al-Qurayyat Governorate, Al-Jouf, and KSA. Those patients were diagnosed by the physician as diabetics based on previous fasting plasma glucose $>126 \mathrm{mg} / \mathrm{dl}$, postprandial blood glucose $>200 \mathrm{mg} / \mathrm{dl}$, and who were receiving oral hypoglycemic agents and/or insulin, and also the patients who were diagnosed as type 1 diabetics and receive insulin injections. Medical data records of the patients were carefully and thoroughly examined. The parameters investigated in this study included age, body mass index (BMI) [BMI=weight in $\mathrm{Kgs}$ divided by height in meters squared], fasting blood sugar (FBS), post-prandial blood sugar (PPBS), glycosylated hemoglobin ( $\mathrm{HbAl} \mathrm{C})$, free tri-iodothyronine (ff3), free thyroxine (fT4) and thyroid-stimulating hormone (TSH). Depending on the existence or absence of thyroid dysfunction in our subjects, they were divided into two main groups; the first included euthyroid diabetic patients who had normal thyroid profile (normal levels of ff3, fT4 and TSH), and the second group included diabetic patients with a coexistent Ill. According to their thyroid profiles, the latter were categorized into four groups as follows:

i. Diabetic patients with increased TSH levels and normal fT3 and fT4 levels were considered to have subclinical hypothyroidism.

ii. Diabetic patients with increased TSH levels and decreased fT3 and f14 levels were considered to have clinical hypothyroidism.

iii. Diabetic patients with decreased TSH levels and normal fT3 and fT4 levels were considered to have subclinical hyperthyroidism.

iv. Diabetic patients with decreased TSH levels and increased fT3 and fT4 levels were considered to have clinical hyperthyroidism. ${ }^{11-12}$

\section{Statistical analysis}

Statistical analysis was done using SPSS version 15 for Windows (SPSS Inc, Chicago, IL, USA).

\section{Results}

The current study included data records of 100 type 1 DM (T1DM) patients (52 males and 48 females) and 100 type 2 DM (T2DM) patients (53 males and 47 females) (Figure 1).

(Table 1) (Figure 2) demonstrate the mean age, BMI, FBS, PPBS, HbA1C, fT3, fT4 and TSH in both T1DM and T2DM groups in both genders; males and females. Mean BMI was $>25$ indicating the states of overweight and obesity. FBS, PPBS and $\mathrm{HbA1C}$ indicate confirmed diabetes. fT3, fT4 and TSH were used to classify patients with TD as shown above in materials and methods.
As shown in Table $2 \&$ Figure 3, the total number of euthyroid cases was $138(69 \%)$ and the total number of diabetic patients with TD was $62(31 \%)$. The most prevalence was found among patients with subclinical hypothyroidism $(25 \%)$, followed by patients with clinical hypothyroidism $(3.5 \%)$ and then patients with clinical hyperthyroidism $(2.5 \%)$ while subclinical hyperthyroidism was not detected:

A. In T1DM group, $67 \%$ of the cases were euthyroid and $33 \%$ had TD. The highest prevalence of TD cases was of subclinical hypothyroidism $(27 \%)$ which was followed by clinical hypothyroidism (4\%), and the third was clinical hyperthyroidism $(2 \%)$. None of the cases had clinical hyperthyroidism.

B. In T2DM group, $71 \%$ of the cases were euthyroid and $29 \%$ had TD. The highest prevalence of TD was of subclinical hypothyroidism $(23 \%)$ followed by clinical hypothyroidism and clinical hyperthyroidism, both of which had the same prevalence $(3 \%)$. None of the cases had clinical hyperthyroidism (Figure 4).

Table 3 \& Figure 5 indicate the gender distribution of thyroid function among the two groups of diabetic patients; T1DM and T2DM. In diabetic patients with subclinical hypothyroidism, there was a highly significant positive correlation between FBS and HbA1 $\mathrm{C}$ ( $p$ value: 0.006 ). Also, a significant positive correlation was found between PPBS and FBS ( $p$ value: 0.046 ). In addition, a significant positive correlation was found between fT3 and FBS (0.035) and a significant positive correlation was found between fT4 and FBS ( $p$ value: 0.017$)$. Non-significant correlations were found between TSH and both of FBS and HbA lc.

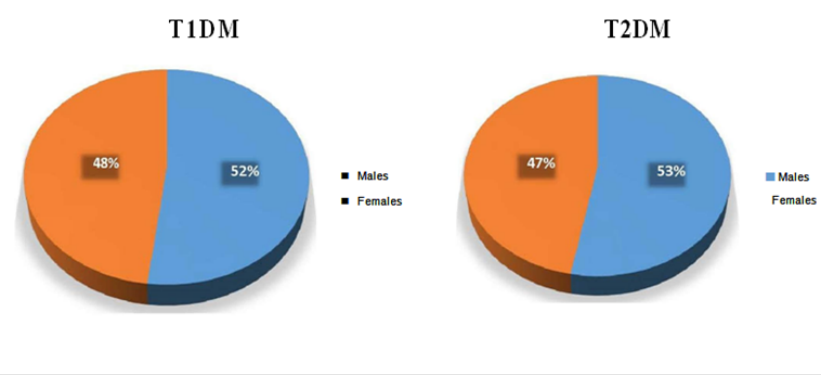

Figure I Sex wise distribution of diabetes among patients.

Table I Parameters Measured in Diabetic Patients

\begin{tabular}{lllll}
\hline Parameters & \multicolumn{1}{c}{ TIDM } & & T2DM \\
\cline { 2 - 5 } & Males & Females & Males & Females \\
\hline Mean age (years) & 46.13 & 43.54 & 52.38 & 50.23 \\
Mean BM $\left(\mathrm{Kg} / \mathrm{m}^{2}\right)$ & 29.62 & 33.0333. & 31.29 & 33.45 \\
Mean HbAlc (\%) & 10.27 & 10.25 & 7.95 & 8.93 \\
Mean FBS (mg/di) & $212.7 \mathrm{I}$ & 225.65225. & 175.29 & 172.52 \\
Mean PPBS (mg/di) & 345.56 & $-1-$ & 272.5 & $246.1 \mathrm{I}$ \\
Mean fT3 (Pmol/L) & 4.76 & 4.7 & 5.14 & 5.36 \\
Mean fT4 (Pmol/L) & $(\mathrm{I}) ;$ & 16.23 & 16.3 & 17.13 \\
Mean TSH (tlU/mlg) & 6.37 & 4.76 & 5.13 & 4.04 \\
\hline
\end{tabular}




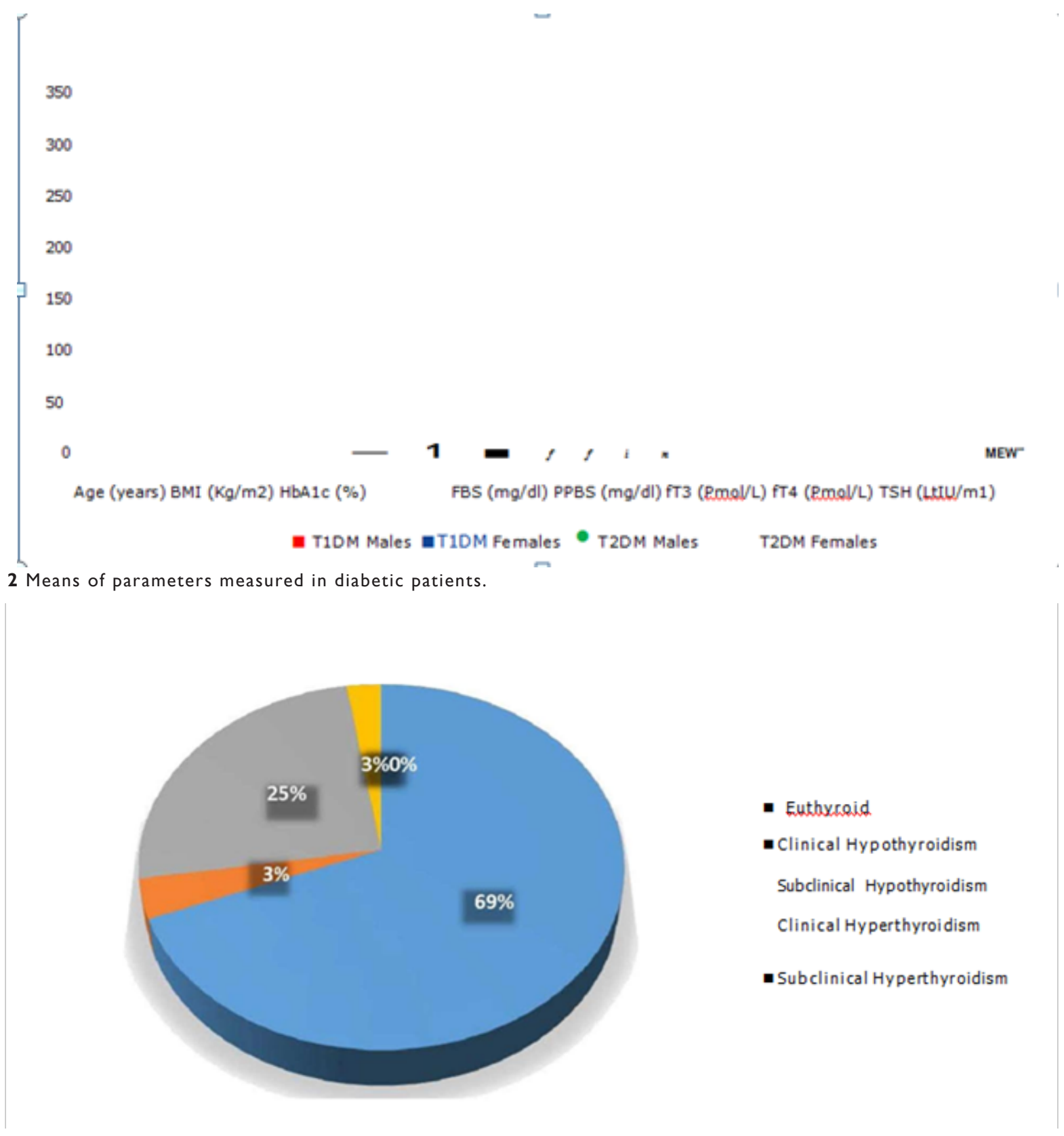

Figure 3 Percentage of each thyroid function in diabetic patients.

Table 2 Thyroid Function in Diabetic Patients

\begin{tabular}{llllll}
\hline Type of DM & Euthyroi & Clinical hypothyroidism & Subclinical hypothyroidism & Clinical hypothyroidism & $\begin{array}{l}\text { Subclinical } \\
\text { hypothyroidism }\end{array}$ \\
\hline TIDM & 67 & 4 & 27 & 2 & 0 \\
T2DM & 71 & 3 & $2-\mathrm{Dec}$ & 3 & 0 \\
Total & 138 & 7 & 50 & 5 & 0 \\
Percent & $69 \%$ & $31 \%$ & & & \\
\hline
\end{tabular}


Table 3 Gender Distribution of Thyroid Function among Diabetic Patients

\begin{tabular}{|c|c|c|c|c|c|c|c|c|c|c|}
\hline \multirow{2}{*}{$\begin{array}{l}\text { Type of } \\
\text { DM }\end{array}$} & \multicolumn{2}{|c|}{ Euthyroid } & \multicolumn{2}{|c|}{ Clinical hypothyroidism } & \multicolumn{2}{|c|}{$\begin{array}{l}\text { Subclinical } \\
\text { hypothyroidism }\end{array}$} & \multicolumn{2}{|c|}{$\begin{array}{l}\text { Clinical } \\
\text { hypothyroidism }\end{array}$} & \multicolumn{2}{|c|}{$\begin{array}{l}\text { Subclinical } \\
\text { hypothyroidism }\end{array}$} \\
\hline & Males & Females & Males & Females & Males & Females & Males & Females & Males & Females \\
\hline TIDM & 36 & 31 & 2 & 2 & 14 & 13 & 0 & 2 & 0 & 0 \\
\hline T2DM & 39 & 32 & I & 2 & 13 & 10 & 0 & 3 & 0 & 0 \\
\hline Total & 75 & 63 & 3 & 4 & 27 & 23 & 0 & 5 & 0 & 0 \\
\hline
\end{tabular}
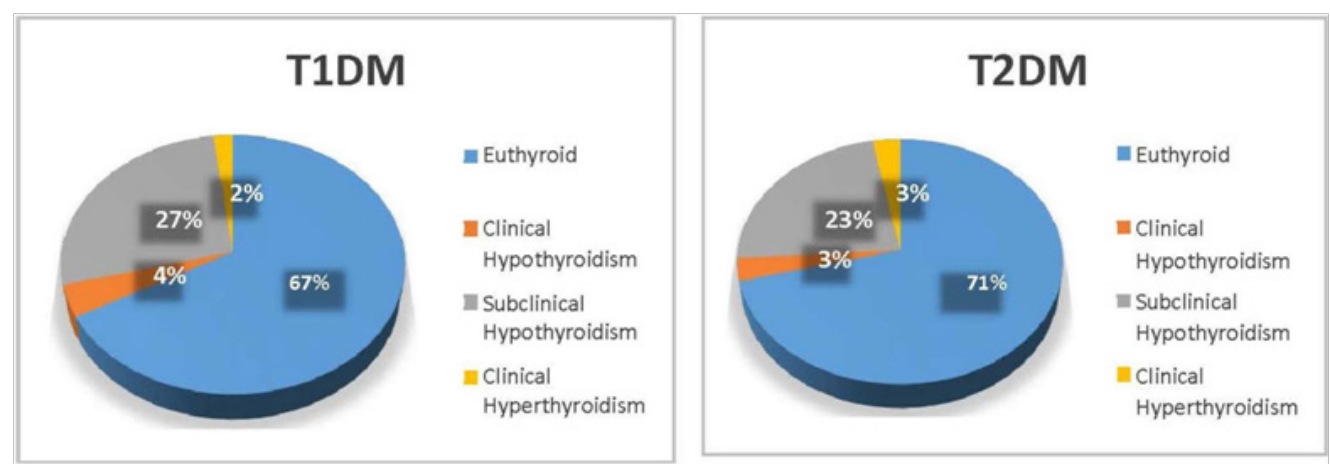

Figure 4 Thyroid function in TIDM and T2DM patients.

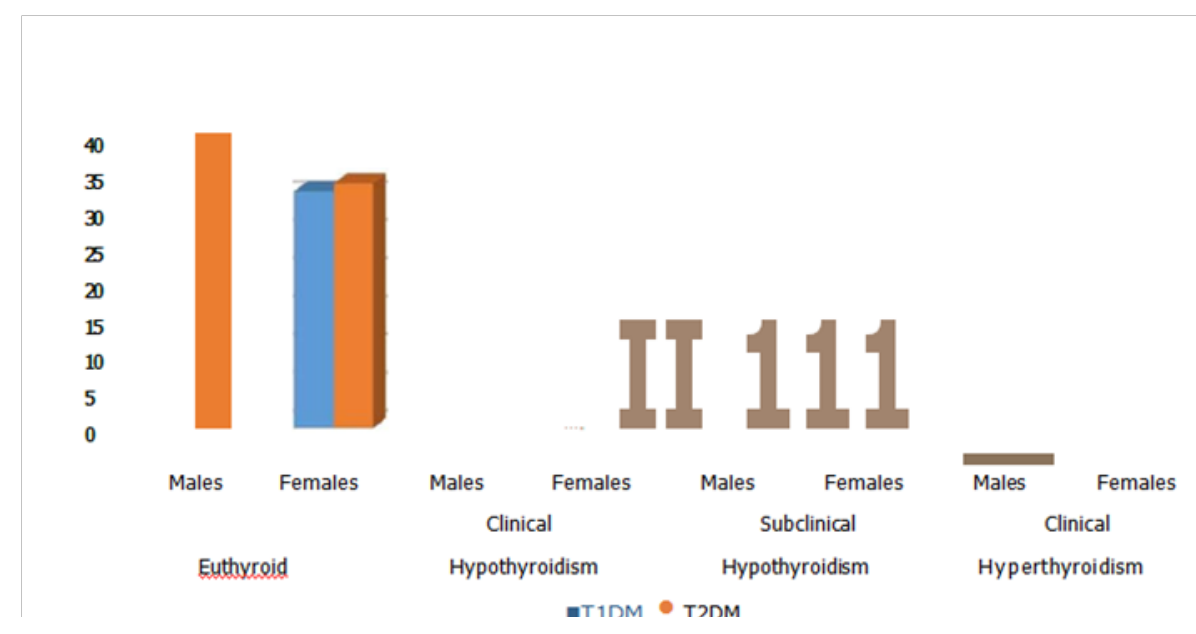

Figure 5 Gender distribution of thyroid function among diabetic patients.

\section{Discussion}

Diabetic patients have a higher prevalence of TD (hyperthyroidism and hypothyroidism) compared with the normal people because patients with one organ-specific autoimmune disease usually are likely to develop other autoimmune disorders. ${ }^{13}$ Triiodothyronine (T3), thyroxine (T4) and thyroid-stimulating hormone (TSH) are the three hormones measured to diagnose thyroid dysfunction. In the present study, out of the two hundred diabetic patients, 138(69\%) patients were found to have normal thyroid functions (euthyroid) and $62(31 \%)$. Low patients had thyroid disorder. This means that almost one third of the diabetic subjects in our study had TD. This outcome is in line with other studies which found that the overall prevalence of TD among diabetic subjects was $12.3 \%,{ }^{14} 12.7 \%,{ }^{15} 12.5 \%,{ }^{16}$ and
13.4\%). ${ }^{17}$ Moreover, a study found that $46.5 \%$ of patients with DM had abnormal thyroid profile. ${ }^{18}$ Furthermore, a study carried out in Kingdom of Saudi Arabia on patients with T2DM found that $16 \%$ of them had TD. ${ }^{19}$

In the current study, the total number of diabetic patients with TD was $62(31 \%), 50$ patients of them had subclinical hypothyroidism (25\% of the total number) this means that subclinical hypothyroidism was the most prevalent TD in our patients. This is in concurrence with the results of other studies. ${ }^{15,16,20-23}$ In the present study the prevalence of TD was $37 \%$ in female and $25 \%$ in male diabetic patients, similar results were found in another study. ${ }^{1}$ Both of Thyroid hormones and insulin antagonize each other and they affect metabolism in body cells, any change of one of them directly or indirectly affects the other. ${ }^{24}$ 
DM disturbs thyroid function by affecting both the hypothalamic control of TSH production and the transformation of T4 to T3 in the peripheral tissue. ${ }^{20}$

The increased TSH level in diabetic patients may be a result of medications they receive which suppress the level of fT4 and T4, while raise levels of TSH, ${ }^{25}$ another cause is insulin which raises T4 level while suppresses T3 level by inhibiting hepatic conversion of T4 to $\mathrm{T} 3{ }^{26}$ a third reason may be autoimmune diseases, ${ }^{27}$ and fourthly is the prevalence of thyroid antibodies in patients with DM..$^{28}$ Moreover, the increased levels of TSH in DM may result from the presence of thyroid hormone binding inhibitor (inhibitor of $\mathrm{T} 4$ to 33 conversion), dysfunction in hypothalamopituitary thyroid axis and the impact of poor control of diabetes on thyroid hormone concentration. ${ }^{29}$ Furthermore, since TD and DM have a common autoimmune etiology, increased TSH in diabetic patients may be caused by immunological disturbances. ${ }^{7}$ Thyroid Association guidelines for type 2 diabetes mellitus patients recommended testing of thyroid profile at the age of thirty-five, and then every five years. ${ }^{5}$ Moreover, The American Association of Clinical endocrinologists, Thyroid Disease Clinical Practice Guidelines recommended investigation of TSH in diagnosis, ${ }^{30}$ furthermore, The British Thyroid Association and the Association of Clinical Biochemistry Guidelines recommended analyzing of thyroid function tests and antibodies for diabetic patients in pregnancy. ${ }^{5}$

\section{Conclusion}

Subclinical hypothyroidism is the most prevalent thyroid dysfunction in our diabetic patients and its most important consequence is high possibility of progression to clinical hypothyroidism. It may increase the risk of cardiovascular disease in diabetic patients though inter-relationships with dyslipidemia, insulin resistance, and vascular endothelial dysfunction.

\section{Recommendations}

Measuring thyroid profile should be set as a protocol in clinical settings for regular screening of thyroid abnormalities in all diabetic patients, which will allow early treatment of subclinical hypothyroidism and decrease morbidity and mortality associated with coexistence of TD in diabetic patients.

\section{Acknowledgements}

I would like to express the deepest appreciation to my supervisor Dr. Ibrahim Elmenshawi, Diabetes and internal medicine specialist and Head of Insulin pump unit in QDC. I'm so thankful for his aspiring guidance, valuably constructive criticism and friendly advice during this work and for his sincere guidance in writing this research paper. Also, I would like to show my gratitude to Dr. Sultan Saoud Alotaibi, Consultant of Diabtes and The Head of Diabetes Centre in Al-Qurayyat for giving me a good guideline for getting patients' data records. In addition, dealing with data records of the patients.

\section{Conflict of interest}

Author declares that there is no conflict of interest.

\section{References}

1. Jain G, Marwaha TS, Khurana A, et al. Prevalence of thyroid disorders in patients of type 2 diabetes mellitus. IJMDS. 2013;2(2).

2. Hilton CW, Mizuma H, Svec F, et al. Relationship between plasma Clyco (His-Pro), a neuropeptide common to processed protein foods and $\mathrm{C}$ peptide/ insulin molar ratio in obese women. Nutr Neurosci. 2001;4(6):469-474
3. Wild S, Roglic G, Green A, et al. Global prevalence of diabetes. Diabetes Care. 2004;27(5):1047-1053.

4. American Diabetes Association. The expert committee on the diagnosis and classification of diabetes mellitus. Diabetes Care. 2002;25(1):S3-20.

5. Nicholas A. Type 2 diabetes mellitus and thyroid dysfunction: an intertwined duo. African Journal of Diabetes Medicine. 2015;22(2)

6. American diabetes association. Diagnosis and classification of diabetes mellitus. Diabetes care. 2010;33(Supp 1):S62-S69.

7. Bhavthankar S, Madole M, Somwanshi S, et al. Evaluation of Thyroid Hormones in Patients with Type II Diabetes Mellitus. Journal of Medical Education \& Research. 2013;3(2).

8. Vondra K, Vrbikova J, Dvorakova K. Thyroid gland diseases in adult patients with diabetes mellitus. Minerva Endocrinol. $2005 ; 30(4): 217-236$.

9. Jeri RR, Stephen FW. Hyperthyroidism: Diagnosis and Treatment. Am Fam Physician. 2005;72(4):623-630

10. Vanderpump MP. The epidemiology of thyroid disease. Br Med Bull. 2011;99:39-51

11. Vikhe VB, Kanitkar SA, Tamakuwala KK, et al. Thyroid dysfunction in patients with type 2 diabetes mellitus at tertiary care centre. Nat J Med Res. 2013;3(4):377-380.

12. Palma CC, Pavesi M, Nogueira VG, et al. Prevalence of thyroid dysfunction in patients with diabetes mellitus. Diabetol Metab Syndr. 2013;5(1):58

13. Practica Wu. Thyroid Disease and Diabetes. Clinical diabetes. 2000;18(1):38-39.

14. Papazafiropoulou A, Sotiropoulos A, Kokolaki A, et al. Prevalence of thyroid dysfunction among greek type 2diabetic patients attending an outpatient clinic. J Clin Med Res. 2010;2(2):75-78.

15. Nobre EL, Jorge Z, Pratas S, et al. Profile of the thyroid functions in a population with type-2 diabetes mellitus. Endocrine Abstracts. 2008;3:298.

16. Radaiedeh AR, Nusier MK, Amari FL, et al. Thyroid dysfunction in patients with type 2 diabetes mellitus in Jordan. Saudi Med J. 2004;25(8):1046-1050.

17. Perros P, McCrimmon RJ, Shaw G, et al. Frequency of thyroid dysfunction in diabetic patients: value of annual screening. Diabet Med. 1995;12(7):622-627.

18. Udiong CEJ, Udoh AE, Etukudoh ME. Evaluation of Thyroid function in Diabetes mellitus in Calabar, Nigeria. Indian $J$ Clin Biochem. 2007;22(2):74-78.

19. Akbar DH, Ahmed MM, Al-Mughales J. Thyroid dysfunction and thyroid autoimmunity in Saudi type 2 diabetics. Acta Diabetol. 2006;43(1):14-18.

20. Nandyala V, Gandiah P, Sivarajappa P, et al. Thyroid Disorders in Type 2 Diabetes Mellitus. International Journal of Recent Trends in Science and Technology. 2013;9(2):250-255.

21. Babu K, Kakar A, Byotra SP. Prevalence of thyroid disorder in type II diabetes mellitus patients. J Assoc Phys Ind. 2001;49:43.

22. Chubb SA, Davis WA, Inman Z. Prevalence and progression of subclinical hypothyroidism in women with type 2 diabetes: the Fremantle Diabetes Study. Clin Endocrinol. 2005;62(4):480-486

23. Guang RY, Jin KY, Lin Z, et al. Association between subclinial hypothyroidism and proliferative diabetic retinopathy in type 2 diabetic patients: A case control study. Tohoku J Exp Med. 2010;222(4):303-310.

24. Sugure DD, Mc Evoy M, Drury MI. Thyroid disease in diabetics. Postgrad Med J. 1999;680-684. 
25. Carreras-Gonzalez G, Perez A. Thyroid autoimmunity at onset of type 1 diabetes as a predictor of thyroid dysfunction. Diabetes Care. 2007;30(11):e121.

26. Suzuki Y, Nanno M, Gemma R, et al. The mechanism of thyroid hormone abnormalities in patients with diabetes mellitus. Nihon Naibunpi Gakkai Zasshi. 1994;70(4):465-470.

27. Reusch CE, Tomsa K. Serum fructosamine concentration in cats with overt hyperthyroidism. J Am Vet Med Assoc. 1999;215(9):1297-1300.
28. Baron DN. Hypothyroidism and Diabetes Mellitus. Lancet. 1955;386:796-800a.

29. Celani MF, Bonati ME, Stucci N. Prevalence of abnormal thyrotropin concentrations measured by a sensitive assay in patients with type 2 diabetes mellitus. Diabetes Res. 1994;27(1):15-25.

30. Baskin HJ, Cobin RH, Duick DS, et al. American Association of Clinical Endocrinologists Medical guidelines for clinical practice for the evaluation and treatment of hyperthyroidism and hypothyroidism. Endocr Pract. 2002;8(6):457-469. 